\section{SARS-COV-2 E PEDIATRIA: RIORGANIZZARE LE MODALITÀ DI LAVORO E LA COMUNICAZIONE, MA COME?}

L'importante lavoro dei pediatri di famiglia (PdF) della bergamasca sul vissuto clinico e assistenziale dell'infezione da SARSCoV-2 nel territorio italiano più colpito dalla pandemia' (in sintesi riportato a pag. 465) pone molte riflessioni che vanno oltre, come gli Autori del lavoro giustamente sottolineano, quello che è stato il contesto di assistenza nei mesi terribili che sono stati affrontati dai PdF (come dai medici di Medicina generale $M M G$ ) oltre che da quelli ospedalieri.

Di fatto è oramai chiaro che l'infezione ha colpito relativamente poco l'età pediatrica e adolescenziale ${ }^{2,3}$ e che l'effetto secondario della pandemia, in estrema sintesi, è stata la riduzione (in diversi casi dimostratamente rischiosa) degli accessi nei Pronto Soccorso (PS) pediatrici, ${ }^{4,5}$, ma anche del numero delle visite e dei controlli ambulatoriali, come la ricerca dimostra in modo atteso, ma con una documentazione epidemiologica chiara e inequivocabile'.

Gli Autori del lavoro evidenziano che le forme di assistenza sono cambiate, con un maggiore accesso alla comunicazione online (messaggi, e-mail, video). Aspetti che si sono resi evidenti anche in contesti più specialistici della Pediatria, come quelli deputati alla cura dei bambini e delle famiglie con bisogni speciali $^{6}$. Non sappiamo quanto queste modalità di assistenza corrispondano a una altrettanto efficace qualità percepita e documentata negli indicatori di salute, tra cui anche il vissuto familiare che deriva da questa esperienza. Quello che sappiamo è che l'uso di messaggi, e-mail, non rappresenta un sistema compiuto di "relazione". Occorre avere affinato competenze a riguardo e strutturato una relazione di cura; la comunicazione esclusivamente scritta, senza queste, potrebbe risultare impositiva, in quanto si tratterebbe di un ascolto/lettura passiva delle informazioni ricevute.

In molti dicono che quanto è successo dovrà portare necessariamente a una riorganizzazione dell'assistenza. È verosimile che molte visite e controlli nella Pediatria ambulatoriale e ospedaliera (come accessi di PS) siano inutili o ridondanti, ma si tratterebbe di capire allora quali dovrebbero essere le funzioni complessive dei servizi deputati alle Cure primarie. I bilanci di salute sono un caposaldo dell'assistenza, ma rivolto a quali obiettivi? Siamo sicuri che la ridondanza di alcune informazioni (rivolte ad esempio alla prevenzione, ai prime mille giorni di vita, a supporto della cronicità, per riportare solo alcuni esempi) non sia necessaria per un migliore impatto utile per raggiungere determinati risultati? Aspetto difficile da esplicitare e documentare, ma è noto che i processi di comunicazione nella loro complessità hanno tante variabili di cui tenere conto: aspetti verbali ma anche non verbali che determino di fatto una "relazione" ${ }^{\prime \prime}$. La costruzione di una relazione di buona qualità presuppone innanzitutto una modalità di rapporto nella quale le parti coinvolte sono disposte a costruire insieme qualcosa, condividendo quello che sono, che provano, che sanno e pensano relativamente a ciò che le ha portate a incontrarsi, pur tenendo conto dei rispettivi ruoli e responsabilità. Questo significa che le parti si riconoscono, concedendo l'una all'altra il rispetto e la considerazione che desiderano a loro volta ricevere ${ }^{7}$, e che nel caso della Pediatria possono far prendere le decisioni migliori per il benessere di neonati, bambini e adolescenti.

A nostro arviso, volendo sottolineare solo alcuni degli aspetti potenziali di una nuova modalità di assistenza, resta da chiedersi se "la differenziazione di ruoli e funzioni" all'interno delle singole realtà assistenziali e nella famosa integrazione ospedale- territorio non possano davvero essere messe in conto come indubbie potenzialità. Per riportare alcuni esempi: nell'ambito della Pediatria di gruppo non può essere immaginato che la teleassistenza, integrata a quella di prossimità, sia appannaggio di gruppi professionali che dedicano parte delle loro attività a svolgere queste funzioni? Ad esempio sui pazienti cronici, con una democratica, partecipe e utile diffusione di strumenti tecnologici e valutativi? Essendo consapevoli che un'assistenza di questo tipo può correre il rischio di creare problemi di inadempienze, disparità e marginalizzazione che andrebbero puntualmente verificati ${ }^{8}$. E non si potrebbe immaginare che le figure infermieristiche (che sono state scarsamente utilizzate dai PdF in questo periodo come la ricerca documenta chiaramente)' possano trovare un ruolo, da sempre auspicato e mai realizzato in pieno, come figure case-manager della complessità di determinate categorie di pazienti ad alto carico assistenziale che risulterebbero essere "facili" da individuare? ?, 10. Il case management ha una lunga storia di forte impatto sulla tradizione dei Servizi sanitari esteri, e in Italia questa storia è decisamente più recente e poco sperimentata in ambito pediatrico.

Certo, non basta la semplice comunicazione a distanza (anche se utile nel capire i bisogni), ma se si immagina una professionalizzazione di questo modello di assistenza si tratterebbe di capire a chi è affidato, con quali modalità e corresponsabilità e come debba integrarsi con un progetto di cura fatto di necessaria prossimità.

Gli esperti dell'assistenza integrata ci dicono che i modelli organizzativi più funzionali sono quelli a rete, o per gruppi di lavoro, che garantirebbero una possibile risposta efficace per superare le debolezze dell'organizzazione piramidale-gerarchica o di singoli professionisti separati dal contesto, permettendo di: a) accelerare i flussi informativi; $\mathbf{b}$ ) ridurre il peso della burocratizzazione; c) decentrare compiti e responsabilità, aumentando flessibilità e dinamismo dei sistemi; d) garantire integrazione e multidisciplinarietà di conoscenze, competenze, tecniche e pratiche; e) creare forti relazioni personali e professionali per favorire il raggiungimento degli obiettivi. Ma per fare questo occorre scommettere su formazione, capacità, competenza e verifiche puntuali, superando ruoli e funzioni assegnate in modo burocratico o strettamente contrattuale. Saremo capaci di farlo, visto che tutti pensiamo che quanto si è verificato con la pandemia non potrà che vederci diversi rispetto a prima? Ovviamente in meglio, si spera.

Oggi la sensazione diffusa è che gli imprevisti che si sono verificati dovuti al lockdown possono avere generato un nuovo modo del prendersi "cura", ma che risulterebbe in ogni caso parziale o incompleto se non vissuto come una vera progettualità. Si potrebbe pensare che destinare del tempo per creare una relazione e comunicare con il paziente o con i colleghi sia "tempo sprecato", in un'attività in cui il carico di lavoro è sempre più oneroso e la corsa "contro il tempo" è di fatto uno dei problemi più frequenti e scoraggianti. Tuttavia, ove la comunicazione sia di qualità, sostenuta cioè da solide competenze relazionali, rappresenta, al contrario, un pilastro fondamentale per la pratica professionale e per l'erogazione di un progetto di cura efficace, appropriato, sicuro ed efficiente. Non è un caso che il Codice Deontologico della professione medica e infermieristica affermi che "Il tempo della comunicazione (va considerato) quale tempo di cura".

A questo, a tutto questo, sarà in qualche modo impossibile rinunciare. Si tratterebbe ora di capire quali possano essere i migliori modelli di organizzazione di rete. Tenendo in considerazione che all'assioma "non esiste un'assistenza qualificata 
ospedaliera senza una qualificata assistenza territoriale e viceversa" è arrivato il tempo di dare un contenuto organizzativo, diffuso, partecipe e di cui rendere conto. Con la prospettiva di chi ha ancora tanto da fare rispetto a ciò che è stato già fatto: caratteristica che non dipende dagli anni che passano, ma di chi resta sempre con lo sguardo rivolto in alto e di chi ha messo il piede sul primo piolo della scala. II dibattito è aperto.

\section{Bibliografia}

1. Bonicelli I, Carrozzo R, Bacchini M, et al. SARS-CoV-2 e Pediatria territoriale: Ármageddon o stimolo per riorganizzare il nostro modo di lavorare? Medico e Bambino Pagine Elettroniche 2020;23(7): 174-9. 2. Parri N, Magistà AM, Marchetti $F$, et al. Characteristic of Covid-19 infection in pediatric patients: early findings from two Italian Pediatric Research Networks. Eur J Pediatr 2020;179(8): 1315-23.

3. Garazzino S, Montagnani C, Donà D, et al. Multicentre Italian study of SARS-CoV-2 infection in children and adolescents, preliminary data as at 10 April 2020. Euro Surveill 2020;25(18):2000600.

4. Ciofi Degli Atti ML, Campana A, Muda AO, et al. Facing SARS-CoV2 pandemic at a Covid-19 regional children's hospital in ltaly. Pediatr Infect Dis J 2020;39(9):e22 1-5.

5. Cella A, Marchetti F, lughetti L, et al. Italian Covid-19 epidemic: effects on paediatric emergency attendance - a survey in the Emilia Romagna region. BM Paediatr Open 2020;4(1):e000742.

6. Taiè $S$, Canali $G$, Russo $M$, et al. La disabilità al tempo del Covid19. Medico e Bambino pagine elettroniche 2020;23(26): 143-5.

7. Ministero della Salute. Comunicazione e perfomance professionale: metodi e strumenti. Elementi teorici della comunicazione. Maggio 2015. 8. Michelle W. Katzow MW, Steinway C, Jan S. Telemedicine and health disparities during Covid-19. Pediatrics 2020;146(2): Oe20201586

9. Nardini C. II valore irrinunciabile dell'infermiere. Medico e Bambino $2019 ; 38(8): 539-40$.

10. Ciofi D Ciolini G. Cosa ci ha insegnato il COVID-19. La voce degli infermieri. Pediatria 2020;10(7-8):24-5.

Federico Marchetti

\section{OBESITÀ NELL'ETÀ EVOLUTIVA: OLTRE LO STIGMA}

L'eccesso di peso dei bambini è un problema di salute ormai evidente anche a insegnanti e genitori, oltre che ai medici: in adolescenza un ragazzo italiano su cinque è in sovrappeso o con obesità, secondo i dati della sorveglianza HBSC (Health Behaviour in School-aged Children - https://www.epicentro.iss. it/ hbsc/indagine-2018). Il problema è diventato così frequente che non vi si dà più peso. I nostri occhi si sono così abituati all'eccesso ponderale che questo è realmente percepito solo quando si superano certi livelli o scatta la derisione; eppure gli studi clinici suggeriscono che quasi il $50 \%$ dei bambini con obesità ha un'ipertensione, il $29 \%$ una dislipidemia, il $44 \%$ una steatosi epatica e il 74\% eccede del $5 \%$ il grasso dei muscoli. Quasi tutti questi bambini sono dunque a rischio di gravi complicanze cardiovascolari, endocrinologiche, ortopediche e psicologiche che possono essere manifeste già in età pediatrica: è pertanto necessario eseguire interventi precoci di prevenzione e di cura. L'Ufficio Regionale per l'Europa della World Health Organization (WHO) ha recentemente pubblicato un report sulle azioni e anche sulle mancanze dei vari Stati nell'applicazione dei suoi progetti di contenimento all'obesità, evidenziando anche quanto sia scarso l'interesse generale per migliorare la situazione'. In effetti, anche le segnalazioni che si ricevono da varie zone del nostro Paese sono sconfortanti: chiusura degli ambulatori dedicati all'obesità, per trasferimento o pensionamento del responsabile (e conseguente carenza di personale), mancato o molto ritardato rinnovo di borse di studio per costituire e mantenere team dedicati, che avevano garantito cure efficienti ed efficaci.
L'attenzione giustamente riservata a patologie affini per percorsi diagnostici terapeutici e assistenziali (PDTA) e investimenti (quali diabete o anoressia) sembra svanire per una condizione nettamente più prevalente come l'obesità. Delusione, sfiducia e demotivazioni sono la naturale conseguenza di queste situazioni sia nei pazienti che nei professionisti più impegnati. Sembra che la classe medica, che pure affronta da sempre con coraggio qualunque malattia, anche considerata inguaribile, di fronte a questo tema si sia arresa. In particolare, non si notano diffuse iniziative di aggiornamento per proporre terapie "non giudicanti" e atteggiamenti anti-stigma, come suggeriscono le recenti linee guida europee per le Cure primarie ${ }^{2}$.

Le prevenzioni primaria e secondaria dell'obesità dovrebbero essere di competenza del medico di Medicina generale (MMG) e del pediatra di famiglia (PdF) ${ }^{3}$. Ciò presuppone un processo di formazione iniziale e successivo sostegno da parte degli specialisti. Questo aspetto è di particolare importanza perché negli ultimi anni è maturato a livello internazionale un nuovo modello di approccio che si basa su alcuni punti fondamentali tra cui:

1. Passare da obiettivi irrealizzabili di guarigione a obiettivi condivisibili di miglioramento della salute globale (se ne parla da lungo tempo, ma il messaggio non sembra recepito) ${ }^{4}$.

2. Evidenziare e prevenire lo stigma diffuso sul peso (di questo aspetto si parla sempre più insistentemente e universalmente solo da alcuni anni) $)^{5}$.

La derisione sul peso, sostenuta dalla ricerca di una magrezza irreale, a simboleggiare emozioni e sogni più affascinanti della dura e povera realtà, promossa da movimenti artistici della fine del XIX secolo dopo la nascita della fotografia, sta crescendo e prevalendo su quella relativa a una appartenenza etnica, religiosa e sessuale. Lo stigma non favorisce una sana consapevolezza del peso e quindi una crescita della motivazione alla cura, ma aumenta depressione e ansia, riduce l'autostima e la self-efficacy, crea vergogna, che isola e paralizza. Insomma ostacola la cura, togliendo a curanti e curati la sensazione di potercela fare.

Nel tentativo di aumentare il livello di allerta generale sul tema obesità, il WHO sembra piuttosto favorire lo stigma senza riuscire a migliorare l'offerta di cure.

Finora le strategie proposte ai professionisti sanitari per l'obesità sono state "l'educazione nutrizionale dei bambini" per prevenire e "la prescrizione di una buona dieta" per curare. Ricercatori esperti e adolescenti in rete ci allertano che ciò non solo è insufficiente, ma potenzialmente pericoloso.

L'educazione nutrizionale è un campo che conosciamo ancora poco. "Educare" è una parola cui spesso si danno significati troppo riduttivi: non è "far conoscere e apprendere le azioni giuste, dare regole precise su campi così ampi e personali come cosa mangiare e quanto muoversi", ma "far scoprire come scegliere il meglio per se stessi e sentirsi bene".

Curare con la prescrizione e la dieta senza modificare I'ambiente familiare è fallimentare. Educare bambini e adolescenti è assolutamente insufficiente, è invece necessaria un'educazione delle famiglie, perché tutti si sostengano vicendevolmente nel cambiamento ${ }^{7}$. Nell'adolescenza, periodo cui si dovrebbe favorire lo sviluppo all'autonomia, pensare di intervenire con prescrizioni è un controsenso ${ }^{8}$.

La famiglia ci prova e fallisce, il pediatra ci prova e fallisce, lo specialista o addirittura il team ci provano e falliscono. Questa catena di fallimenti crea sensi di colpa che alla fine si scaricano sui bambini/ragazzi. Deriderli con commenti sul corpo o rim- 
proveri sui comportamenti, anche se fatti a fin di bene, procura un danno enorme. La derisione extra-familiare nell'infanzia si dimentica, quella dei genitori no. Frasi come: "Lo sai che tu questo non lo puoi mangiare!", "Non vedi che i pantaloni non ti stanno piư!", "Questo l'ho comprato solo per tuo fratello!" certamente non fanno star bene i ragazzi, non li aiutano a crescere e non migliorano i loro comportamenti alimentari. In adolescenza affermazioni sulla dieta o "sapere di avere una mamma a dieta"9 favoriscono comportamenti alimentari insani (introdurre poche calorie, per esempio meno di 900 al giorno, con pasti ipocalorici pronti o saltare i pasti) o altamente pericolosi (digiunare, vomitare e usare farmaci per dimagrire) arrivando a causare disturbi del comportamento alimentare (DCA).

Lo stigma su corpo e peso, con tutte le sue conseguenze negative, attualmente adottato in modo continuo e precoce da tutti, viene potenziato da fenomeni di introiezione e nuoce prima e più delle pur numerose e ben note complicanze organiche dell'obesità $^{10}$. Proprio i genitori già stigmatizzati sul peso sono quelli che usano parlare ai bambini e ragazzi in modo da perpetuare lo stigma e favorirne la sua introiezione ${ }^{1}$. Purtroppo anche i medici e i pediatri cadono frequentemente nella trappola della derisione ${ }^{12}$. Sentendosi impotenti di fronte all'obesità, cercano di ottenere un cambiamento colpevolizzando i genitori e richiamando le possibili complicanze cardiache e metaboliche a cui espongono i loro figli. Ma se una svolta si realizza, non è quella che si vorrebbe. Infatti, la derisione e la colpevolizzazione riducono l'autostima e l'autoefficacia, causano tristezza e isolamento, aumentano il "mangiare per emozioni" e le perdite di controllo alimentare.

Eppure l'alternativa esiste: ritrovare la misura negli obiettivi e fare da modello, condividendo le difficoltà ed evitando la derisione. Questo metodo dovrebbe pertanto essere adottato da genitori, insegnanti, politici, pediatri e da tutti gli altri operatori sanitari, anziché farsi intrappolare dal circolo vizioso che dal fallimento terapeutico porta allo stigma, al rifiuto di una formazione professionale aggiornata e quindi alla negazione di cure adeguate. Per essere operativi, i Centri sopravvissuti aderenti al Gruppo di Studio sull'Obesità della Società Italiana di Endocrinologia e Diabetologia Pediatrica (SIEPD) hanno proposto un questionario da far compilare ai genitori e agli adolescenti su come chiamare questa malattia per motivarli e non deriderli' ${ }^{13}$. Moltissime e inattese sono state le adesioni da ogni parte d'Italia!

In sintesi, 10 mosse vincenti che possono essere adottate dai Sistemi Sanitari ${ }^{14}$ :

1. Partire da una valutazione accurata della situazione ponderale fatta dal pediatra con le carte del WHO.

2. Non parlare di peso né di chilogrammi né di peso o BMI ideale, né all'inizio né durante il percorso e spostare l'obiettivo dal peso e dal corpo alla buona salute e alla socialità, insomma alla qualità della vita.

3. Fare da modello e cercare di migliorare lo stile di vita alimentare e motorio delle famiglie a piccoli passi.

4. Evitare le diete: unico obiettivo in ambito nutrizionale sia la vera dieta mediterranea (mangiare con parsimonia a orari regolari, preferire frutta fresca, verdura, semi, più olio di oliva, bere acqua, ridurre carni rosse e cibi dolci, e muoversi con piacere). Lo stile di vita sano e attivo fa bene a tutti, di ogni peso ed età.

5. Consigliare l'uso di un contapassi per valutare l'attività fisica e aumentarla piacevolmente. Gestire/ridurre l'uso de- gli strumenti elettronici per tutti i familiari, proteggendo l'orario dei pasti e il momento di andare a letto.

6. Proteggere la qualità e quantità del sonno.

7. Cercare eventuali episodi di derisione in famiglia e a scuola e cercare soluzioni per ridurli.

8. Costruire percorsi educativi di miglioramento centrati sull'empowerment delle famiglie e sulla comunicazione non deridente con la famiglia e fra i suoi membri, per aumentare la loro auto-efficacia, curando la loro autoderisione e vergogna.

9. Sostenere i piccoli risultati raggiunti già nelle intenzioni di cambiare e poi nei cambiamenti dei comportamenti di ciascun familiare. Non cercare risultati immediati.

10. Non sostenere un'eccessiva preoccupazione per il cibo, il peso e le forme e se la si evidenzia, contrastarla.

\section{Bibliografia}

1. World Health Organization Regional Office for Europe. Mapping the health system response to childhood obesity in the WHO European Region. An overview and country perspectives 2019.

2. Durrer Schutz D, Busetto L, Dicker D, et al. European practical and patient-centred guidelines for adult obesity management in Primary Care. Obes Facts 2019;12:40-66.

3. Chamay-Weber C, Farpour-Lambert NJ, Saunders Gasser C, Martin $\mathrm{XE}$, Gal C, Maggio AB. Obesity management in adolescents: comparison of a low-intensity face-to-face therapy provided by a trained paediatrician with an intensive multidisciplinary group therapy. Obes Facts 2016;9:112-20.

4. Rudolf MC, Krom AJ, Cole TJ. How good are BMI charts for monitoring children's attempts at obesity reduction? Arch Dis Child 2012;97: $418-22$.

5. Dietz WH, Baur LA, Hall K, et al. Management of obesity: improvement of health-care training and systems for prevention and care. Lancet 2015;385:2521-33.

6. Rees RW, Caird J, Dickson K, Vigurs C, Thomas J. 'It's on your conscience all the time': a systematic review of 'qualitative studies examining views on obesity among young people aged $12-18$ years in the UK. BM Open 2014;4:e004404.

7. Moxley E, Habtzghi D, Klinkhamer N, Wang H, Donnelly S, Dykhuizen J. Prevention and Treatment of Pediatric Obesity; A Strategy Involving Children, Adolescents and the Family for Improved Body Composition. J Pediatr Nurs 2019;45:13-19.

8. Ludwig DS. Weight loss strategies for adolescents: a 14-year-old struggling to lose weight. JAMA 2012;307:498-508.

9. Neumark-Sztainer D, Bauer KW, Friend S, Hannan PJ, Story M, Berge JM. Family weight talk and dieting: how much do they matter for body dissatisfaction and disordered eating behaviors in adolescent girls? $J$ Adolesc Health 2010;47:270-6

10. Puhl RM, Suh Y. Health consequences of weight stigma: implications for obesity prevention and treatment. Curr Obes Rep 2015:4: 182-190.

11. Pearl RL, Wadden TA, Shaw Tronieri J, et al. Sociocultural and Familial Factors Associated with Weight Bias Internalization. Obes Facts 2018;11:157-64.

12. Tanas $R$, Begona BG, Caggese $G$, Baggiani F, Valerio $G$, Corsello $G$. Professional Stigma on Weight in the Pediatric Care in Italy and Andalusia: Recognize it to Successfully Treat Obesity. J Obes Ther 2017; $1: 1$

13. Tanas R, S. Bernasconi S, Marsella M, Corsello G. What's the Name: the weight stigma and the battle against obesity. Commentary submitted to Italian Journal of Pediatrics.

14. Tanas R, Caggese G, Lera R. Il pediatra e l'obesità: riprova e vinci in 5 mosse. Quaderni acp 2020;271:4-11.

\section{Rita Tanas \\ Pediatra endocrinologo, Ferrara Valeria Calcaterra}

Pediatria, IRCCS Policlinico "S. Matteo", Università di Pavia Claudia Veronica Carletti, Gianluca Tornese IRCCS Materno-Infantile "Burlo Garofolo", Trieste Sergio Bernasconi Microbiome Research Hub, Università di Parma 